\title{
Development of FD-SOI Monolithic Pixel Devices for High-Energy Charged Particle Detection
}

\author{
K. Hara, K. Shinsho, T. Ishibashi, Y. Arai, T. Miyoshi, Y. Ikemoto, R. Ichimiya, T. Tsuboyama, T. Kohriki, Y. Yasu, \\ Y. Onuki, Y. Ono, H. Katsurayama, A. Takeda, K. Hanagaki
}

\begin{abstract}
Monolithic pixel devices fabricated with a siliconon-Insulator (SOI) technology are excellent candidates to realize particle detectors of fast response and least material yet simple in fabrication. In our SOI pixel devices the sensitive part is the "handle" wafer, to which we examined high resistive FZ wafers of both p- and n-types together with $\mathrm{CZ}$ wafer of n-type. Full depletion of the FZ wafers is easily achievable for typical thicknesses of 260 to $500 \mu \mathrm{m}$. We thinned these devices to 100 to $50 \mu \mathrm{m}$. The response was evaluated with infrared and red lasers, and in a high energy beam. Irradiation to ${ }^{60} \mathrm{Co} \gamma$ was carried out to verify the radiation tolerance of the devices.
\end{abstract}

\section{INTRODUCTION}

$\mathrm{T}$ HE potentiality of novel monolithic pixel devices utilizing the $0.2-\mu \mathrm{m}$ fully depleted silicon-on-insulator (FD-SOI) technology provided by ROHM Lapis Semiconductor [1] has been intensively explored for various applications including $\gamma / \mathrm{X}$-ray imaging and space applications [2]. The SOI handle wafer is high resistive silicon serving as the sensing part and the top is $40-\mathrm{nm}$ thick silicon of $18 \Omega \mathrm{cm}$, separated by a $200-$ $\mathrm{nm}$ thick buried oxide (BOX) layer, see Fig. 1. Such an SOI wafer is realized by adopting the bonded technique provided by SOITEC [3]. A notable feature is that the resistivity of the sensor part can be selected according to the application requirements.

There are several key issues in developing monolithic pixel devices for high-energy charged particle detection; e.g., least material, full depletion, and radiation tolerance.

- Least material is important for minimizing the multiple scattering. Thinning the sensor is particularly beneficial for the monolithic devices where the sensor itself is the main contributor to the amount of material, and is possible for the devices with least noise. We evaluated the sensor characteristics for the devices thinned to $50 \mu \mathrm{m}$ and $100 \mu \mathrm{m}$. The thinned devices were tested in high energy beams.

Manuscript received November 4, 2011. This work was supported in part by Kakanhi [grant number $21244040 \mathrm{~A}$ ].

K. Hara, K. Shinsho and T. Ishibashi are with Graduate School of Pure and Applied Sciences, University of Tsukuba, Tsukuba, Ibaraki 305-8571 Japan (telephone: +81-29-853-4270, e-mail: hara@px.tsukuba.c.jp).

Y. Arai, T. Miyoshi, Y. Ikemoto, R. Ichimiya, T. Tsuboyama, T. Kohriki and Y. Yasu are with Institute of Particle and Nuclear Studies, High Energy Accelerator Organization (KEK), Tsukuba, Ibaraki 305-0801 Japan.

Y. Onuki, Y. Ono and H. Katsurayama are with Graduate School of Science, Tohoku University, Sendai, Miyagi 980-8579 Japan.

A. Takeda is with School of High Energy Accelerator Science, The Graduate University for Advanced Studies, Tsukuba, Ibaraki 305-0801 Japan.

K. Hanagaki is with Graduate School of Science, Osaka University, Toyonaka, Osaka 560-0043 Japan
- With fully depleted devices, the charge is collectable faster, providing a constant amount for minimum ionizing particles. Fully depleted devices are realized by thinning (Czochralski, $\mathrm{Cz}$ sensors) and adopting high resistive FZ (Float Zone) wafers. We recently succeeded in adopting high resistive FZ wafers together with conventional $\mathrm{Cz}$. While only n-type is available for high resistive $\mathrm{Cz}$, both n-type and p-type are possible for FZ wafers. Fully depleted high resistive FZ wafers have an impact on hard X-ray and $\gamma$-ray imaging for increased detection efficiency.

- Radiation hardness is a rather complicated issue in SOI devices since the device is fully contained in oxide. We have carried out a detailed study on transistor basis characteristics [4]-[6]. Here we present the functionality of the devices irradiated to about $10 \mathrm{kGy}$ following the annual radiation level of $1 \mathrm{kGy} / \mathrm{y}$ evaluated for ILD vertex detector to be located at a radius of $5 \mathrm{~cm}$.

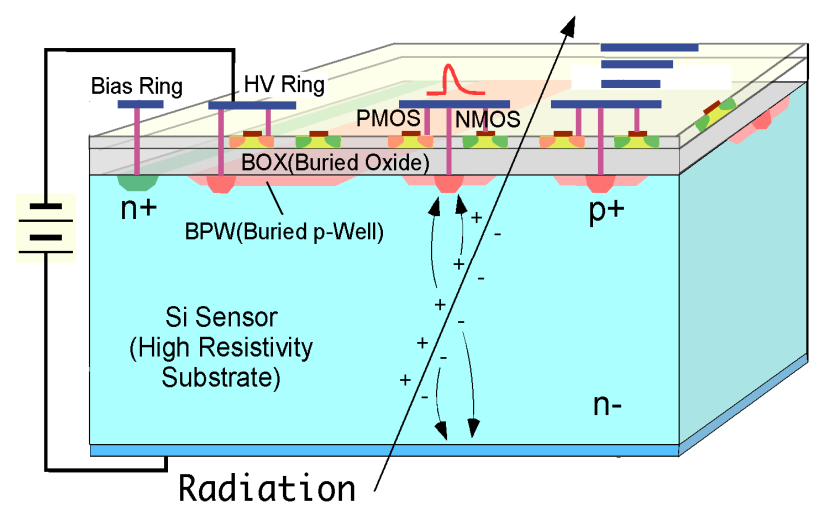

Fig. 1. Schematics of the SOI monolithic pixel device. The pixel electrodes, fabricated through the BOX layer, and SOI MOS transistor terminals are interconnected via metal layers on top of the device. The device is biased from the back or from the front $n^{+}$contact (Bias Ring).

\section{INTPIX3 AND DIPIX2}

The SOI fabrication was carried out on a basis of multi project wafer runs [7], processing various types of sensors on a same 8" wafer. The main process parameters are summarized in Table I. As for the handle wafer, high resistive $\mathrm{Cz}$ (n-type) and FZ (n-type and p-type) are available. The default thicknesses are 260 and $500 \mu \mathrm{m}$, respectively. The FZ wafers are chosen to be rather thick to take full advantage of fully-depleted yet thick devices. The ground back surface was not annealed and found to produce large leakage current if the device is fully depleted and biased from the back though 
aluminum. Therefore no aluminum was added to the FZ devices of the standard thickness.

TABle 1. Main Process Parameters

\begin{tabular}{|c|c|}
\hline process & $\begin{array}{l}0.2 \mu \mathrm{m} \text { low leakage fully depleted SOI CMOS } \\
1 \text { poly }+5 \text { metal layers, MIM }\left(1.5 \mu \mathrm{F} / \mu \mathrm{m}^{2}\right) \text {, DMOS } \\
\text { Core }(\mathrm{I} / \mathrm{O}) \text { voltage }=1.8(3.3) \mathrm{V}, \text { Buried } \mathrm{p} / \mathrm{n}-\text { Well }\end{array}$ \\
\hline SOI wafer & $\begin{array}{l}\text { diameter : } 300 \mathrm{~mm} \phi \\
\text { Top Si : } \mathrm{Cz} \sim 18 \Omega \mathrm{cm} \text { (p-type) }, 40 \mathrm{~nm} \text { thick } \\
\text { Buried oxide: } 200 \mathrm{~nm} \text { thick } \\
\text { Handel wafer: } \mathrm{Cz}(\mathrm{n}, 0.7 \mathrm{k} \Omega \mathrm{cm}), \mathrm{FZ}(\mathrm{n}, 7 \mathrm{k}), \mathrm{FZ}(\mathrm{p}, 40 \mathrm{k})\end{array}$ \\
\hline $\begin{array}{l}\text { Backside/ } \\
\text { thickenss }\end{array}$ & $\begin{array}{l}\text { Cz:thinned to } 260 \mu \mathrm{m} \text {, sputtered with } \mathrm{Al}(200 \mathrm{~nm}) \\
\text { FZ:thinned to } 500 \mu \mathrm{m} \text { (no } \mathrm{Al}) \\
\quad \text { Devices thinned to }(50,100 \mu \mathrm{m}) \text { available }\end{array}$ \\
\hline
\end{tabular}

We evaluated two kinds of integration type devices [8], INTPIX3 and DIPIX2, see Table II. The INTPIX3e chips having an array of $128 \times 128$ pixels of $20 \mu \mathrm{m}$ square are made on $\mathrm{Cz}(\mathrm{n})$ and $\mathrm{FZ}(\mathrm{n})$. The outer chip size is $5 \mathrm{~mm}$ square. Each pixel has an active pixel type circuit integrated, see Fig. 2 . The pixel charges are extracted out of the chip one by one, then digitized by a 12-bit ADC, which is implemented in an SiTCP

TABLE II. CHARATCERISTICS OF DIPIX2 WAFERS AND AVAILABILITY OF THIN INTPIX3.

\begin{tabular}{lccc} 
WAFER TYPE & CZ $(\mathrm{N})$ & FZ $(\mathrm{N})$ & FZ $(\mathrm{P})$ \\
\hline RESISTIVITY $[\mathrm{k} \Omega \mathrm{cm}]$ & 0.7 & 7 & 40 \\
THICK DIPIX2 $[\mu \mathrm{m}]$ & 260 & 500 & 500 \\
FULL DEPLETION $[\mathrm{V}]$ & 340 & 125 & 60 \\
THIN INTPIX3 $[\mu \mathrm{m}]$ & 50,100 & 50,100 & $\mathrm{~N} / \mathrm{A}$ \\
\hline
\end{tabular}

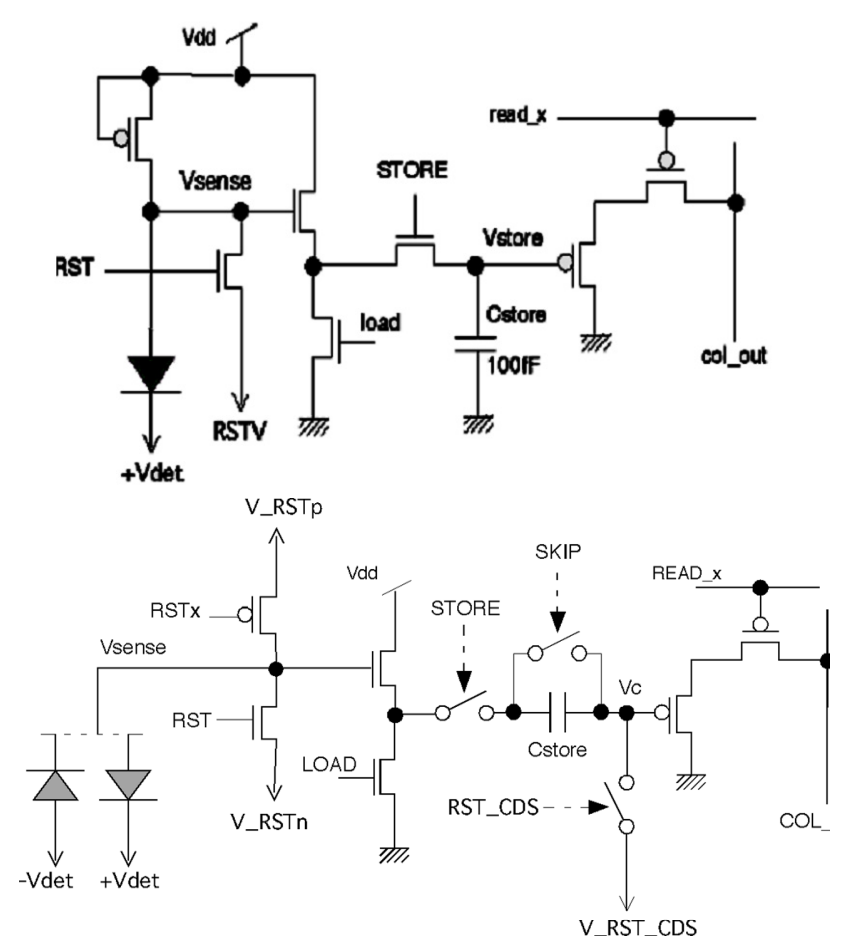

Fig. 2. Diagram of (top) INTPIX3 and (above) DIPIX2 on-pixel circuit. The charge stored in $\mathrm{C}_{\mathrm{STORE}}$ is sent out through the common column line. based readout system, SEABAS [9]. Some of diced sensors were thinned to $50 \mu \mathrm{m}$ or $100 \mu \mathrm{m}$ by NIHON Exceed Co. [10]. Earlier version of INTPIX3a was thinned in a form of wafer by DISCO Japan [11]. The evaluation of the devices thinned by DISCO is reported elsewhere [12].

Since INTPIX3 is a first device where BPW's are integrated, the pixels of INTPIX3a are segmented into eight regions to examine various BPW configurations, including radiation hardness.

The DIPIX2 (Dual Integration type PIXel) was developed in order to read out both n- and p-type signals, as shown in Fig. 2. While other functions like CDS (correlated differential signal) and on-chip signal digitization are implemented, these were set inactive in this study. Therefore the DIPIX2 chips are similar to INTPIX3 chips except that there are $256 \times 256$ pixels of $14 \mu \mathrm{m}$ square.

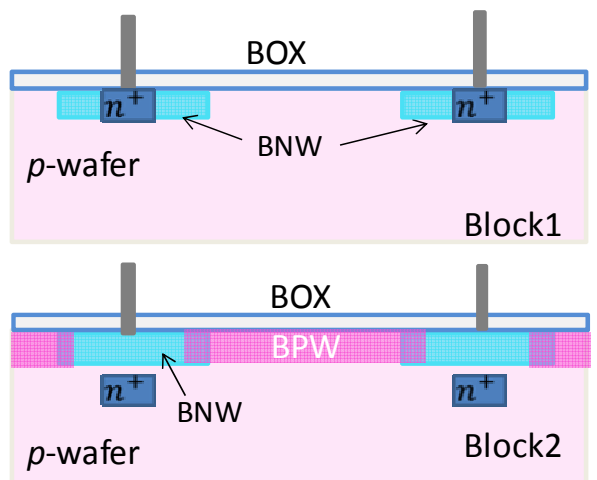

Fig. 3. Configuration of BNW and BPW in the case of p-bulk. All the charge polarities be inverted in the case of n-bulk.

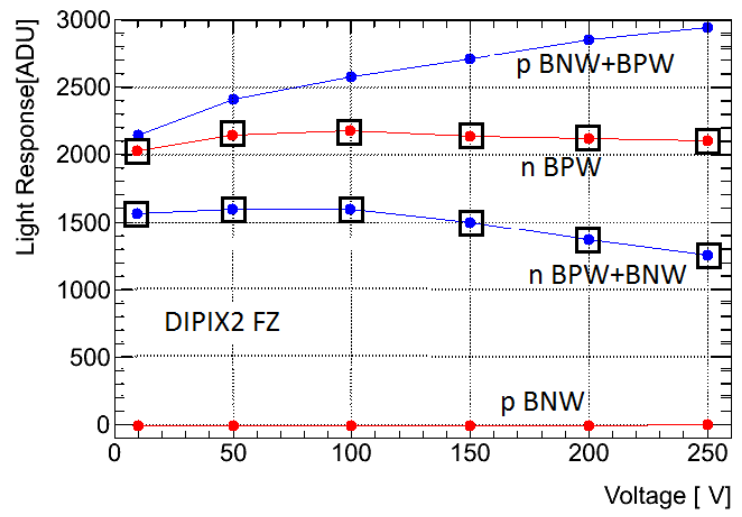

Fig. 4. Response to white light illuminated from the top of DIPIX2 chips. The curves are shown separately for the two blocks of FZ devices, BPW (BNW) only for n-type (p-type), and BPW+BNW.

In the DIPIX2 process, we added BNW for p-type sensor, which corresponds to BPW for n-type sensor. Half of the pixels (Block1) were processed in this way like INTPIX3. The rest half was processed adding both BPW and BNW together, as illustrated in Fig. 3. The BPW (in n) and BNW (in p) are tied to the pixel electrodes; sustaining the nearby potential and suppressing the back-gate effect not deteriorate the electronics performance. This is realized if the added electrodes are wide 
enough while wider electrodes are easier to couple each other. The overlaid electrode, e.g. BPW for p-type, reduces the interpixel coupling, functioning like a p-stop. The effectiveness of additional BPW for the p-type device is evident in Fig. 4 where the response to white light is plotted as a function of the bias. The effectiveness of BNW for the n-type device is not clear from the data.

\section{THINNING}

One of the wafers containing INTPIX3a chips was thinned to $100 \mu \mathrm{m}$ [13] by TAIKO process provided by DISCO Corp. With this process, the outer edge of the wafer is left unground. So thinned wafer is self sustainable, reducing the risk of handling such as at stress relief processing and backside aluminizing. The stress relief process removes the damages and residual strain created at grinding, consisting of dry polishing, chemical mechanical polishing, and wet and dry etchings. Finally the backside was aluminized.

Thinned devices were examined on the full depletion using pulsed lasers. Fig. 5 shows the response of a $100 \mu \mathrm{m}$ device to infrared and red lasers illuminated from the backside of the device [13]. The response to infrared increases with square root of the bias below the full depletion, reaching a constant above. The response to red laser is also consistent that the full depletion is achieved around $90 \mathrm{~V}$.

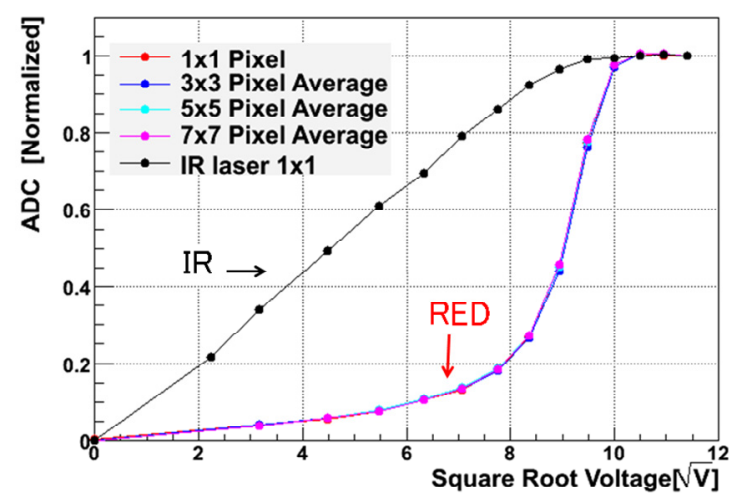

Fig. 5. Response to infrared $(\lambda=1064 \mathrm{~nm})$ and red $(\lambda=634 \mathrm{~nm})$ lasers injected from the backside of a $100 \mu \mathrm{m}$ thick INTPIX3a[13].

Thinning by NIHON Exceed was made on diced INTPIX3e chips from the standard thicknesses. The surface was mechanically and chemically polished using abrasives developed to enable flattening to sub-micron precision. The backside of these devices were not aluminized.

The leakage current of the thinned devices is compared in Fig, 6. Although the leakage current increased by an order of magnitude for FZ devices, the device should be fully depleted already at $5 \mathrm{~V}$ for $100 \mu \mathrm{m}$, showing the increase is in the region above the full depletion. This increase should be attributed to the backside treatment at thinning. For $\mathrm{Cz}$ devices the full depletion is $90 \mathrm{~V}$ for $100 \mu \mathrm{m}$, showing that a contribution exists below full depletion for INTPIX3e. As a comparison, the I-V curve for INTPIX3a did not change by thinning process (by DISCO). We continue to investigate improvement if the devices are thinned by NIHON Exceed on a wafer basis or on a basis of block of chips.

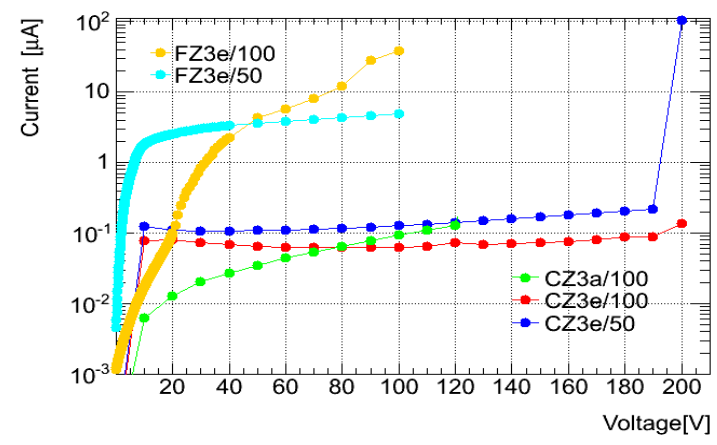

Fig. 6. Leakage currents measured at $20^{\circ} \mathrm{C}$ of thinned INTPIX3. Cz3b was thinned to $100 \mu \mathrm{m}$ on a wafer basis; others are to $50 \mu \mathrm{m}$ or $100 \mu \mathrm{m}$ on a diced chip basis.
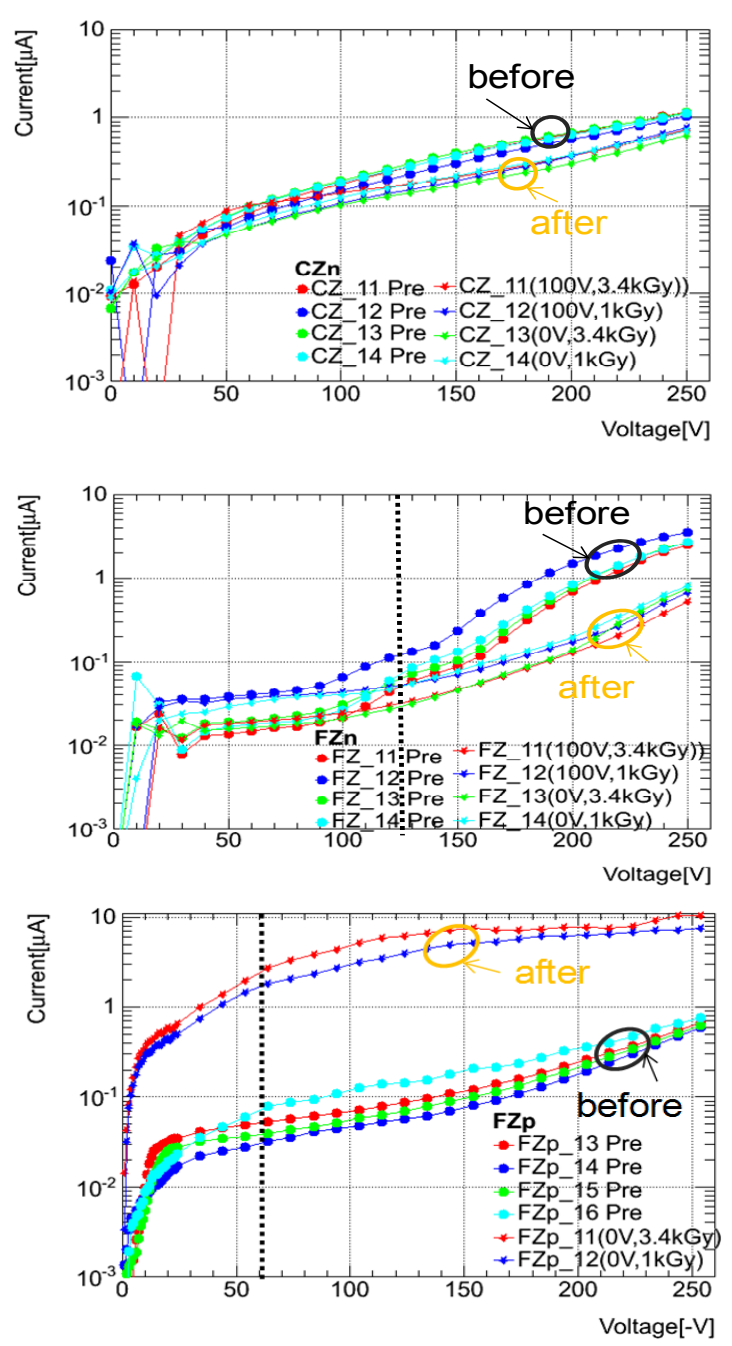

Fig. 7. IV curves of DIPIX2 chips before and after irradiation to $1 \mathrm{kGy}$ and $3.4 \mathrm{kGy}$ : (top) $\mathrm{Cz}$ n, (middle) FZ n, and (above) FZ p. The full depletion voltages are indicated by broken lines for FZ devices, which is $360 \mathrm{~V}$ for $\mathrm{Cz}$ n-type. 


\section{COBALT IRRADIATION}

\section{A. DIPIX2 irradiation}

The DIPIX2 devices were irradiated with ${ }^{60} \mathrm{Co} \gamma$ 's, one group at $1 \mathrm{kGy} / \mathrm{h}$ and another at $3.4 \mathrm{kGy} / \mathrm{h}$. The irradiation was interrupted every $15 \mathrm{~min}$ to evaluate the inter-radiation characteristics and lasted for $1 \mathrm{~h}$ in total. During the irradiation, all the terminals of the chips were ground.

The results are shown in Fig. 7 comparing the IV curves measured at $20^{\circ} \mathrm{C}$ before and after the final irradiation. For the n-type devices both with $\mathrm{Cz}$ and FZ, the leakage current increase is small, or even decreases in the region above the full depletion for the FZ devices. The leakage current increases above the full depletion due to the field lines reaching the backside where many defects were created by thinning. Since there is no aluminum and the silicon surface should be oxidized. A possible interpretation is that the trapped holes created there develop an electron layer at the silicon surface after irradiation. This layer behaves like $\mathrm{n}^{+}$, creating an ohmic contact which acts to reduce the leakage current.

Notable is the nearly two orders of magnitude increase of the leakage current in the FZ p devices. Some front side structure, most likely the bias structure should explain the increase since the leakage starts to increase below full depletion. The primary cause of irradiation is accumulation of holes in the oxide, which attract electrons at the silicon surface. These electrons are usually removed as the depletion develops. The removal is more efficient if the electron layer is near the junction. This preferable situation is realized in the nbulk sensor, where the bias configuration is $\mathrm{p}^{+}-n_{\mathrm{e}}(\mathrm{n})-\mathrm{n}^{+}$, with the electron layer being denoted as $n_{e}$ and (n) being the bulk to be depleted in competition with the electron layer. An explanation for the present observation is that the configuration is $\mathrm{p}^{+}-\mathrm{n}_{\mathrm{e}}(\mathrm{p})-\mathrm{n}^{+}$in $\mathrm{p}$ bulk, where the p-bulk depletion starts at "ohmic" for the electron layer and hence the layer may be intact while the depletion develops. The surface layer depletion is less enhanced for higher resistive devices like our FZ p sensor. Addition of a floating p-stop ring between $\mathrm{p}^{+}$at edge (bias ring) and $\mathrm{n}^{+}$(HV ring) should shut the current flow through the radiation-induced electron layer.

The pixel response was measured in the same system used for pre-irradiation samples. The data shown in Fig. 4 are to be compared with the $1 \mathrm{kGy}$ irradiated data shown in Fig. 8. For n-type devices, the difference between with and without BNW is small. The preference of having both BNW and BPW for the p-type device remains similar, while some response appeared after irradiation for the device without BPW. At a
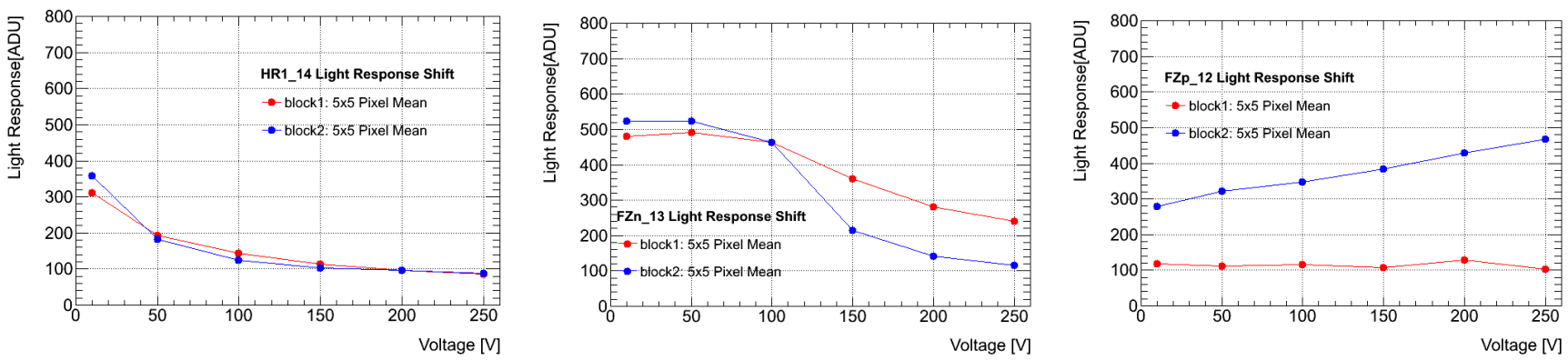

Fig. 8. Response to white light illuminated from the top of DIPIX2 chips, (top) Cz n-type, (middle) FZ n-type, (above) FZ p-type. The curves are shown separately for the two blocks, (red) BPW (BNW) only and (blue) BPW+BNW.
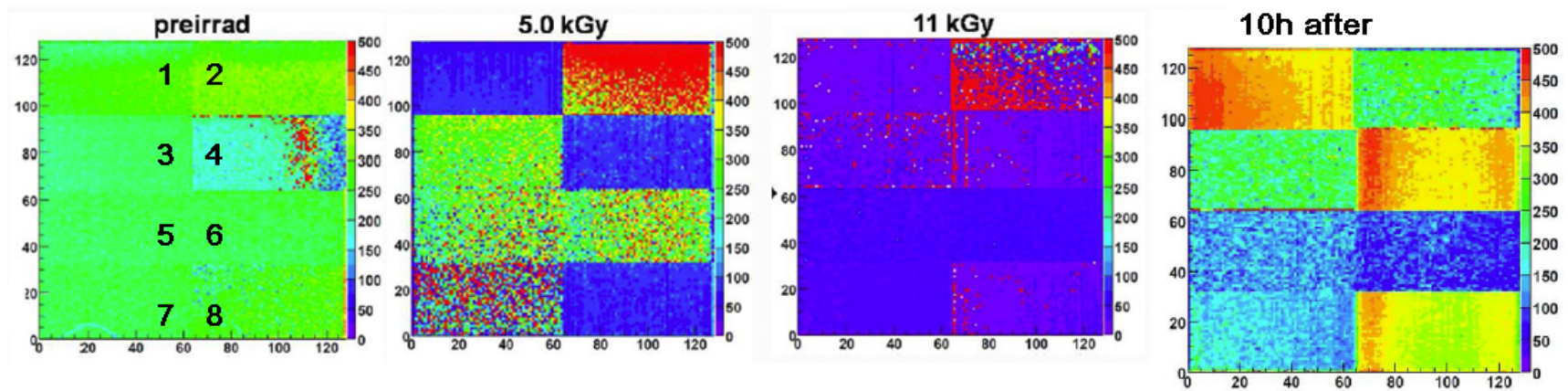
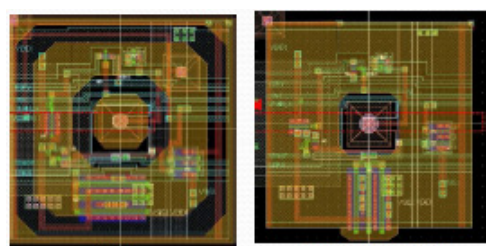

$\mathrm{R} 3(\sim \mathrm{R} 2)$

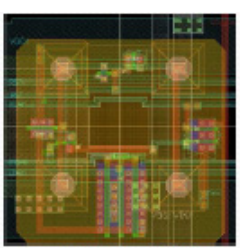

R5 ( R6)

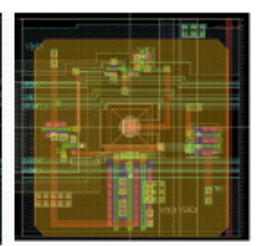

R7 ( DIPIX)
Fig. 9. Response of INTPIX3a to white light at selected dosage. The rightmost is the result $10 \mathrm{~h}$ after $11 \mathrm{kGy}$ irradiation. The BPW structures (yellowish) are shown for typical regions. 
bias of $100 \mathrm{~V}$, the response is reduced to $1 / 15$ for $\mathrm{Cz}-\mathrm{n}, 1 / 5$ for FZ-n, and 1/7 for FZ-p devices.

\section{B. INTPIX3a irradiation}

The signal pulse height is dependent on the BPW/BNW structures since they contribute to the detector capacitance. Also the radiation tolerance is notably dependent on the structure. One INTPIX3a chip, where different BPW structures are implemented, was irradiated at $0.4 \mathrm{kGy} / \mathrm{h}$ to 3.4 $\mathrm{kGy} / \mathrm{h}$ setting all the terminals at ground. The response was examined periodically by illuminating white light uniformly.

As shown in Fig. 9, the response diminished almost completely after $11 \mathrm{kGy}$ where the irradiation was terminated. The device was kept in room temperature $\left(\mathrm{ca} .15^{\circ} \mathrm{C}\right.$ ) for $10 \mathrm{~h}$, then the response of some regions, R2 and R3, recovered to the level of pre-irradiation. The recovery is to compensate for the accelerated irradiation, but the response in some regions remained low. The DIPIX2 chip has single pixel electrode to which $\mathrm{BPW} / \mathrm{BNW}$ is directly connected for n-type/p-type devices such that their potential is controlled by the pixel electrode. Region 7 is similar in configuration to DIPIX2, where the recovery is rather small.

In R3 the BPW potential can be set externally. Setting the BPW floating, or to positive voltage is necessary not to deteriorate the charge collection. The signal collection to charged particles is discussed below.

\section{TEST BEAM}

\section{A. Electron beam}

The INTPIX3a thinned to $100 \mu \mathrm{m}$ was tested in electron beam of $673 \mathrm{MeV}$ provide by $1.2 \mathrm{GeV}$ electron synchrotron of Research Center for Electron and Photon Science, Tohoku University [13]. The cluster charge distributions are compared between the thinned INTPIX3a and $260 \mu \mathrm{m}$ thick INTPIX3b. Although no identical BPW structures are available for INTPIX3a and INTPIX3b, INTPIX3a/R7 and INTPIX3b/R3 are picked up. The structures are similar but the area of BPW in INTPIX3b/R3 is smaller than INTPIX3a/R7.

The MPV of the distribution increases with the bias for the $260 \mu \mathrm{m}$ thick device while it is consistent to be saturated for $100 \mu \mathrm{m}$ one which is fully depleted at $90 \mathrm{~V}$. The relative response at $100 \mathrm{~V}$ resulted in 1.4 , which is consistent with 1.57 that accounts for the different sizes of the BPW and was extracted by a calibration using X-ray [8].

\section{B. Pion beam}

The INTPIX4e devices thinned to 50 and $100 \mu \mathrm{m}$ were tested in high energy pion beam provided by the SPS at CERN. Typical cluster charge distributions at $100 \mathrm{~V}$ for $\mathrm{Cz}$ and at $16 \mathrm{~V}$ for FZ are shown in Fig. 11 where the pedestal spread distributions are also shown. On a pixel basis, the onbeam pedestal spread is calculated as the sigma of a Gaussian fitted around the maximum of the charge distribution. The onbeam pedestal spread turned out to have somewhat wider distributions among the pixels than the off-beam pedestal spread.
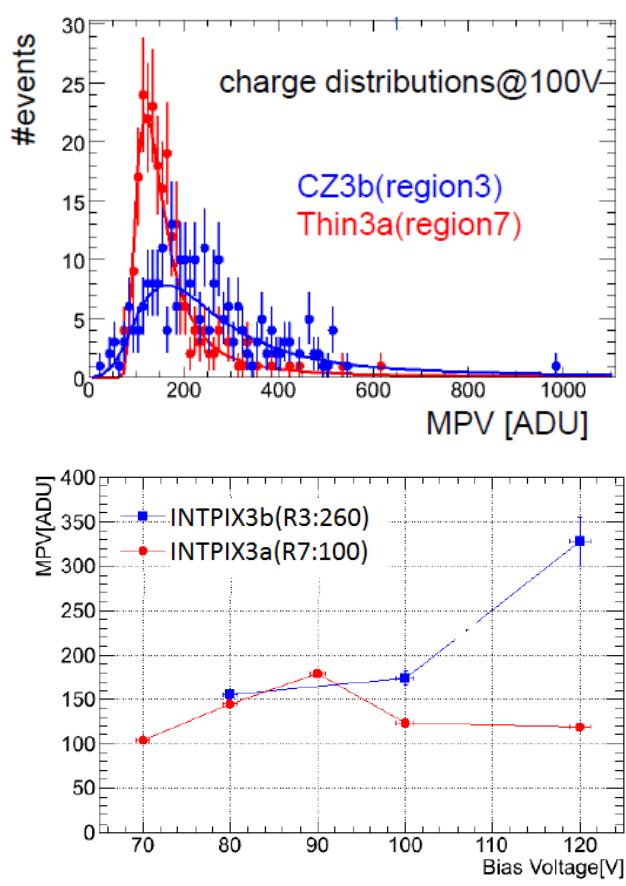

Fig. 10. (top) Cluster pulse height distributions compared between INTPIX3a (R7; $100 \mu \mathrm{m})$ and INTPIX3b (R3; $260 \mu \mathrm{m})$ at $100 \mathrm{~V}$, (above) bias dependence of the most probable values.

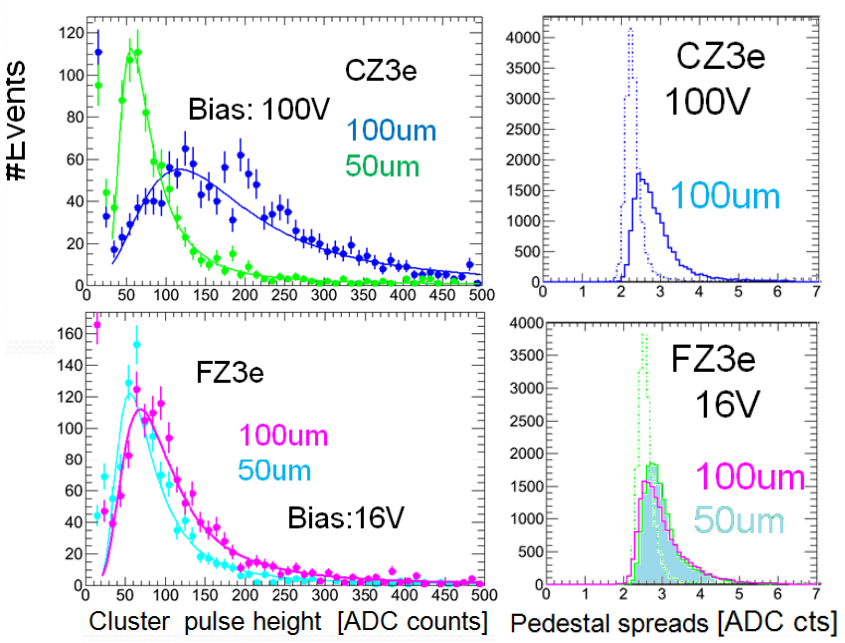

Fig. 11. (left) Collected charge distribution for pions compared between $\mathrm{Cz}$ and FZ INTPIX4e devices of 50 and $100 \mu \mathrm{m}$ thicknesses. (right) The pedestal spread distributions are shown for on (solid) and off (dotted) beam.

The cluster charge distribution peaks corresponding to the charged pions. The MPV's are plotted in Fig. 12 as a function of square-root of the bias for $\mathrm{Cz}$ devices. The data for $\mathrm{FZ}$ devices are available only at $16 \mathrm{~V}$. The ratio of the response of $50 \mu \mathrm{m}$ and of $100 \mu \mathrm{m}$ reflects the thickness ratio for $\mathrm{Cz}$ devices. The response ratio for the FZ is not described by the 
thickness, although the data for $50 \mu \mathrm{m}$ is similar to $\mathrm{Cz}$ of same thickness.

The signal-to-noise ratio is shown in Fig. 13 where the ratio is defined as the MPV of the signal distribution divided by that of the on-beam pedestal spread distribution. $\mathrm{S} / \mathrm{N}$ ratio of about 40 is achievable for $\mathrm{Cz}$ devices of $100 \mu \mathrm{m}$ thickness. Note that the devices were operated at room temperature and the noise can be reduced further by nearly a factor of three by lowering the temperature to $0^{\circ} \mathrm{C}[14]$.

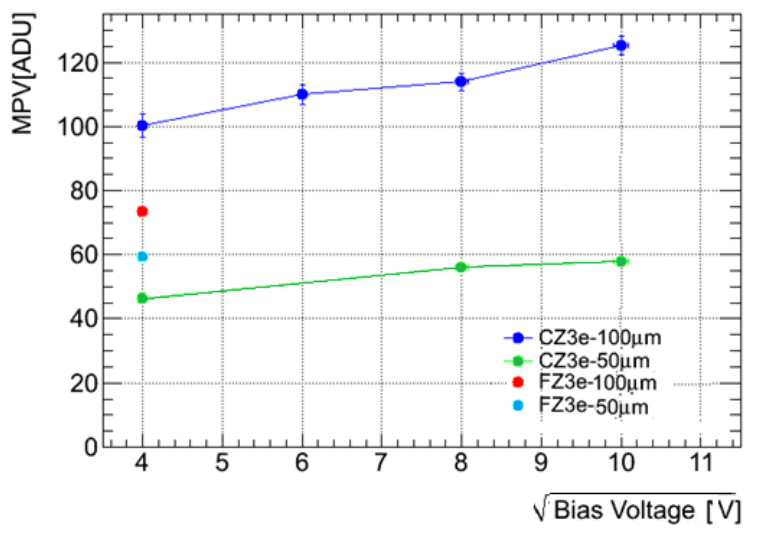

Fig. 12. The MPV of pedestal subtracted distributions as a function of square root of the bias voltage

The relative response of $\mathrm{R} 3$ to $\mathrm{R} 7$ of INTPIX3a was investigated in the CERN beam, which resulted in $1.48 \pm 0.11$. Therefore, as discussed before, the BPW structure of INTPIX3a/R3 is radiation hard and emerges non inferior signal output as DIPIX2 and should be investigated further as radiation hard devices.

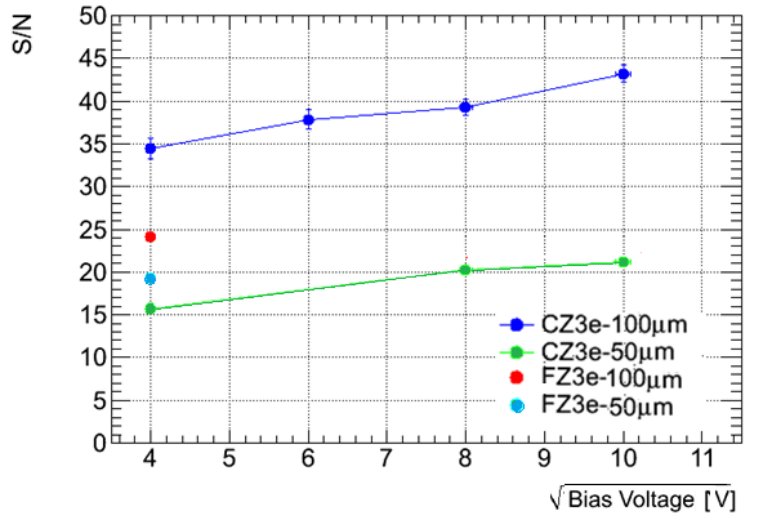

Fig. 13. The signal to noise ratios as a function of square root of the bias voltage.

\section{SUMMARY}

Application of SOI pixel devices to high-energy particle detection is under investigation. Some key technologies such as thinning the devices to $100 \mu \mathrm{m}$ or less and adopting high resistive FZ wafers have been realized successfully. Radiation hardness to $10 \mathrm{kGy}$ and above has been demonstrated while the BPW/BNW designs need to be optimized both for signal collection and radiation hardness. The devices thinned to 100 $\mu \mathrm{m}$ were tested in a high-energy beam, providing a signal-tonoise ratio of 40 at room temperature.

\section{ACKNOWLEDGMENT}

The authors wish to thank S. Kim of University of Tsukuba for his continuing encouragement to this project. This work is supported by KEK Detector Technology Project and also by VLSI Design and Education Center (VDEC), The University of Tokyo, with the collaboration of the Cadence Corporation and Mentor Graphics Corporation.

\section{REFERENCES}

[1] ROHM LAPIS Semiconductor Co., Ltd.: http://www.lapis-semi.com/en/. Formerly named OKI Semiconductor.

[2] T. G. Tsuru et al., "Development and Performance of X-Ray Astronomical SOI Pixel Sensor", presented at this symposium, N8-5; A. Takeda et al., "Test Results of X-Ray Imaging Sensor with SOI CMOS Technology", presented at this symposium, N21-3; S. G. Ryu et al., "Design and Development of Trigger-Driven Readout with X-Ray SOI Pixel Sensor", presented at this symposium, NP3.M-90; S. Nakashima et al., "Development of a Built-in Analog-to-Digital Converter for an XRay Astronomy Detector Using the SOI CMOS Technology", presented at this symposium, NP3.M-92: T. Miyoshi et al., "Performance Study of Monolithic Pixel Detectors Fabricated with FD-SOI Technology", presented at this symposium, NP5.S-164.

[3] SOITEC: http://www.soitec.com/en/technologies/smart-cut/

[4] K. Hara et al., "Development of INTPIX and CNTPIX Silicon-OnInsulator Monolithic Pixel Devices", PoS (Vertex2010) 033.

[5] M. Kochiyama et al., "Radiation effects in silicon-on-insulator transistors with back-gate control method fabricated with OKI Semiconductor 0.20 um FD-SOI technology", Nucl. Instr Meth A636 (2011) S62.

[6] K. Hara et al.'Radiation Resistance of SOI Pixel Devices fabricated with OKI 0.15um FD-SOI Technology." IEEE TNS, Vol.56-5, October 2009, pp. 2896-2904.

[7] See SOIPIX group homepage: http://rd.kek.jp/project/soi/ for MPW information.

[8] Y. Miyoshi et al., "Recent progress of the pixel detectors R\&D based on the SOI technology", talk given at TIPP 2011 Conference, Proceedings to be published in Physics Procedia.

[9] T. Uchida and M. Tanaka, Development of a TCP/IP Processing Hardware, IEEE Nucl. Sci. Symposium, NS33-6, pp1411 - 1414, 2006.

[10] NIHON Exceed Co.: http://www.nihon-exceed.co.jp/indexe.htm

[11] DISCO Co.: http://www.disco.co.jp/eg/solution/library/taiko.html.

[12] K. Shinsho et al., "Evaluation of SOI Monolithic Pixel Devices Thinned to $100 \mu \mathrm{m}$ ", IEEE 2010 NSS CR-N21-3.

[13] Y. Onuki et al., "SOI Developments", talk given at VERTEX 2011, Proceedings to be published in Proceeding of Science.

[14] A. Takeda et al., Presented at this Symposium. See [2]. 